\title{
GRUPOS DE PESQUISA BRASILEIROS COM ÊNFASE NA PANDEMIA DA COVID-19
}

BRAZILIAN RESEARCH GROUPS ABOUT COVID-19 PANDEMIC

Thiago Ferreira de Sousa*a, Sueyla Ferreira da Silva dos Santos ${ }^{b}$, Gildeene Silva Fariasc, Alex Carneiro Brandão ', Ayalla Oliveira Chavese, Fernanda Carneiro Mussif, Marcia Grisottid $^{d}$

\author{
Universidade Federal do Recôncavo da Bahia, Amargosa, BA, Brasila \\ Universidade Federal do Amazonas, Parintins, AM, Brasil \\ Faculdade Estácio, Teresina, PI, Brasilc \\ Universidade Federal de Santa Catarina, Florianópolis, SC, Brasil \\ Instituto Federal de Educação, Ciência e Tecnologia, Uruçuca, BA, Brasile \\ Universidade Federal da Bahia, Salvador, BA, Brasilf \\ *E-mail: tfsousa_thiago@yahoo.com.br
}

\section{RESUMO}

O objetivo deste estudo foi analisar as características dos grupos de pesquisa brasileiros com linhas de pesquisa direcionadas ao estudo da pandemia da COVID-19. Trata-se de estudo transversal e documental, cuja fonte de dados foi o Diretório dos Grupos de Pesquisa no Brasil do Conselho Nacional de Desenvolvimento Científico e Tecnológico (CNPq). A busca foi realizada com base nos descritores "COVID-19", "SARS-CoV-2", "coronavírus" e "pandemia", sem o emprego de filtros. Foram selecionados todos os grupos de pesquisa que apresentaram, no mínimo, uma linha de pesquisa sobre o tema. Do total de 55 grupos de pesquisa identificados, 42 grupos atenderam os critérios na primeira busca realizada no mês de junho, e posteriormente, no mês de julho, outros quatro grupos foram incluídos. Os grupos de pesquisas sobre a COVID-19 concentram-se na região sudeste. Nas áreas temáticas observou-se o predomínio das Ciências da Saúde e Ciências Biológicas, prevalecendo as linhas de pesquisas sobre estudos de diagnóstico, informação e comunicação, tratamento farmacológico, epidemiologia, e economia e saúde ocupacional. Conclui-se que houve o aumento dos grupos de pesquisa ativos que apresentavam, no mínimo, uma linha de pesquisa destinada a investigação da pandemia da COVID-19, com ênfase em conteúdos relacionados ao tratamento e diagnóstico da doença.

Palavras-chave: Epidemias; Pesquisa; Infecções por Coronavírus.

\section{ABSTRACT}

This study was to analyze the characteristics of Brazilian research groups with lines of research aimed at studying the pandemic of COVID-19. Carried out a cross-sectional and documentary study, whose data source was the Directory of Research Groups in Brazil of the National Council for Scientific and Technological Development (CNPq). The search was performed based on the descriptors "COVID-19", "SARS-CoV-2", "coronavirus" and "pandemic", without the use of filters. All research groups that presented at least one line of research on the topic were selected. Of the 55 research groups identified, 42 groups met the criteria in the first search carried out in June, subsequently, in the search in July, another four groups were included. Research groups on COVID-19 are concentrated in the southeast region. In the thematic areas, there was a predominance of Health Sciences and Biological Sciences, with lines of research about diagnostics, information and communication, pharmacological treatment, epidemiology, and economics and occupational health. It is concluded that there was an increase in active research groups that had at least one line of research aimed at investigating the pandemic of COVID-19, with an emphasis on content related to the treatment and diagnosis of the disease.

Keyword: Epidemics; Research; Coronavirus Infections. 


\section{INTRODUÇÃO}

O relato dos primeiros casos do novo coronavírus (COVID-19) em humanos ocorreu em dezembro de 2019 na cidade de Wuhan, na China (WANG et al., 2020). O aumento do número de pessoas hospitalizadas com diagnóstico de pneumonia de etiologia desconhecida fez com que o governo chinês reportasse a Organização Mundial da Saúde (OMS) o surto endêmico. Após análise de sequenciamento genético, identificou-se o surgimento do novo coronavírus (SARS-Cov-2), causador da síndrome respiratória aguda grave (ZHU et al., 2020).

Desde janeiro de 2020, o surto epidemiológico da COVID-19 alcançou uma escala global com registro de casos notificados fora da China em todos os continentes (OPAS, 2020), com a chegada ao Brasil em 25 de fevereiro de 2020, na cidade de São Paulo (BRASIL, 2020). A OMS declarou que a COVID-19 constituía Emergência de Saúde Pública de Importância Internacional (ESPII), e logo em seguida decretou a pandemia em 1 de março de 2020 (OPAS, 2020).

A alarmante situação global de saúde direcionou esforços políticos, econômicos e científicos na busca de estratégias de controle e redução dos danos provocados pela pandemia. Alianças globais foram fortalecidas para o seu enfrentamento, com destaque para a COVID-19 "Clinical Research Coalition" (CATTANI, 2020) e a rede "Solidarity" lançada pela OMS para garantir celeridade na avaliação dos estudos sobre a eficácia de tratamentos e vacinas para controle da pandemia (WHO, 2020). Todavia, pesquisas nacionais alertam para o movimento de contramão da atuação internacional do Brasil dada a ausência ou divergência de decisões políticas relacionadas a mudanças climáticas e as medidas preventivas para controle da propagação da COVID-19 (VENTURA et al., 2020; NUNES, 2020).

No Brasil, apesar da tendência drástica de redução orçamentaria dos investimentos em Ciência e Tecnologia, a qual atingiu $56,36 \%$ do orçamento entre 2013 e 2019 (MACÁRIO; REIS, 2020) e do desprestígio da educação e ciência pela sociedade e política brasileira (VENTURA et al., 2020), é importante ressaltar que as universidades e os serviços de saúde pública são o sustentáculo para o combate à COVID-19 no país, sendo os principais responsáveis pela assistência à saúde e fomento às pesquisas sobre a temática (MOTA; FERREIRA; LEAL, 2020; RIBEIRO et al., 2020).

Mesmo com o (des)incentivo à pesquisa, o Brasil esteve, em 2019 , na $17^{a}$ posição no ranking global de países com publicações científicas sobre o novo coronavírus (FAPESP, 2020), bem como tem histórico de protagonismo em estudos relacionados a doenças tropicais e virais, como a Zika (VENTURA et al., 2020). Os estudos de intervenção brasileiros, em sua maioria, estão sendo desenvolvidos por universidades e hospitais públicos que tem investigado protocolos de tratamento com o uso de células tronco, dexametasona, cloroquina ou hidroxicloroquina (monoterapia ou associada a azitromicina) como opções terapêuticas para a COVID-19 (RIBEIRO et al., 2020).

Diante da gravidade da pandemia e da ausência de informações prévias sobre a condução eficiente e eficaz do tratamento, nota-se a necessidade emergencial de investigação sobre os diferentes fatores relacionados a COVID-19 e seus impactos globais. Nesse sentido, os grupos de pesquisa representam o eixo central do direcionamento e implementação das pesquisas para o enfrentamento da COVID-19 e de seus efeitos nas dimensões sociais, ambientais, econômicas, políticas e de saúde coletiva.

O conhecimento sobre o estado atual da produção científica e sobre as características dos grupos de pesquisa brasileiros permitem compreender e analisar o caminho que a ciência brasileira está seguindo sobre determinada área (MENDONÇA et al., 2017; SCHMITT; BERTOLDI; MAZO, 2017; DIAS et al., 2020; CHAVES; CRUZ, 2017). Diante disso, é possível estabelecer um olhar diferenciado sobre as diversas questões que envolvem os estudos relacionados à pandemia e aos centros que se destacam na condução das investigações. Mediante o exposto, o objetivo deste estudo foi analisar as características dos grupos de pesquisa brasileiros com linhas de pesquisa direcionadas a investigação da pandemia da COVID-19.

\section{MATERIAL E MÉTODOS}

Este estudo é de caráter descritivo e documental e foi realizado por meio da análise das informações eletrônicas sobre os grupos de pesquisa cadastrados no Diretório de Grupos de Pesquisa do Conselho Nacional de Pesquisa e Desenvolvimento Científico (CNPq), por meio do link: http://dgp.cnpq.br/dgp/faces/consulta/consulta_parame trizada.jsf. Realizou-se a busca no dia 10 de junho de 2020 utilizando-se os seguintes descritores: "COVID19", "SARS-CoV-2", "coronavírus" e "pandemia", sem o emprego de filtros. No dia 03 de julho de 2020 foi repetida a busca utilizando os mesmos critérios da busca inicial de forma a identificar outros grupos de pesquisa.

Os grupos que apresentaram, no mínimo, uma linha de pesquisa ou que nos objetivos da linha ou palavras-chaves descreviam o foco na pandemia da 
COVID-19 foram incluídos. Dos grupos selecionados foram identificados o nome do grupo, se estavam ativos ou inativos, a data da última atualização, a região geográfica de localização, o tipo de instituição de vínculo (pública e privada), o ano de formação, a área e subárea, a quantidade de pesquisadores registrados (professores, técnicos e estudantes), a titulação do líder, a quantidade de linhas totais e as específicas para a pandemia da COVID-19 e os objetivos das linhas de pesquisa específicas.

Os dados foram inseridos no software Excel (versão 2017) e analisados em médias e desvio padrão (DP), frequências absolutas e relativas. Os objetivos das linhas de pesquisa relacionadas a pandemia foram analisados por meio da técnica de análise de conteúdo (BARDIN, 2002).

\section{RESULTADOS}

Na busca inicial, foram encontrados 55 grupos de pesquisa cadastrados no $\mathrm{CNPq}$, sendo 33 identificados pelo termo COVID-19, 10 pelo termo coronavírus e 15 pelo emprego do termo pandemia.
Com a utilização do termoSARS-Cov-2 não foram encontrados grupos de pesquisa. Por fim, permaneceram para análise 42 grupos de pesquisa, sendo 33 localizados pelo termo COVID-19, cinco por coronavírus e quatro por pandemia. Os grupos excluídos $(\mathrm{N}=13)$ ocorreram, pois, foram repetidos entre os termos. Com a realização da segunda busca, foram encontrados mais quatro grupos, sendo três por meio do termo COVID-19 e um grupo via termo SARS-COV-2. A amostra final deste estudo foi de 46 grupos.

Todos os grupos selecionados estavam ativos. O grupo com tempo de atualização mais antigoocorreu em 31 de março de 2020 e, o mais recente, em 3 de julho de 2020. Todos os líderes informaram título de doutor, com exceção de três grupos cuja informação não constava. Em relação a quantidade de pesquisadores vinculados, as médias foram: 12,93 ( $\mathrm{DP}=13,27)$ professores pesquisadores (total de 595); 10,17 $(\mathrm{DP}=13,80)$ estudantes pesquisadores (total de 468); $0,65(\mathrm{DP}=1,67)$ de técnicos pesquisadores (total de 30). Dentre os grupos selecionados, $15(32,61 \%)$ foram formados em 2020 e, o mais antigo, foi formado em 1969 (Figura 1).

Figura 1. Descrição do ano de formação dos grupos de pesquisa que estudam a pandemia da COVID-19. Brasil, 2020.

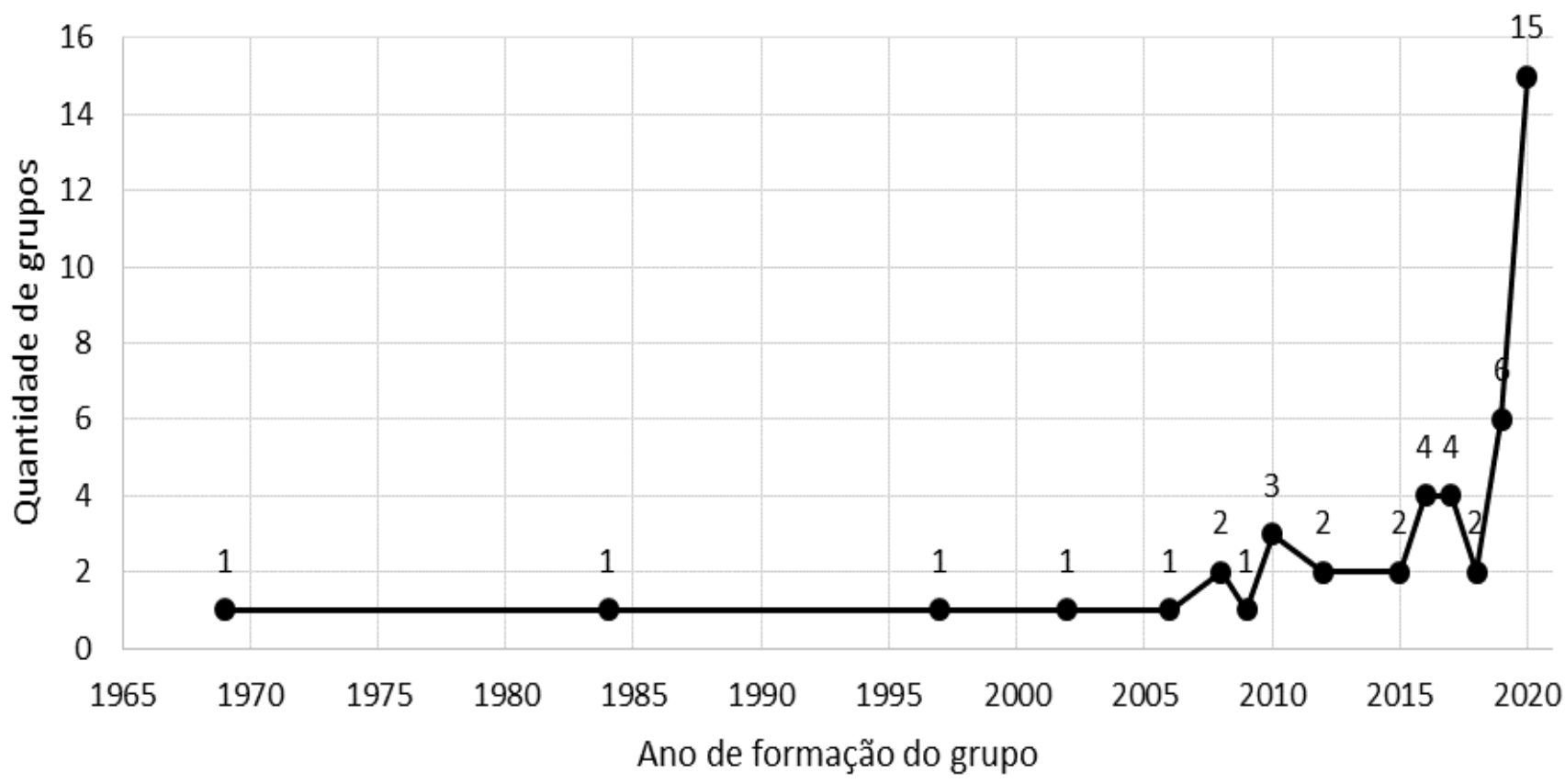


Na Tabela 1 é apresentada a descrição dos grupos de pesquisa em relação as regiões geográficas brasileiras.
Houve predomínio para a região sudeste $(45,65 \%)$, seguido da região nordeste $(23,91 \%)$.

Tabela 1. Descrição dos grupos de pesquisa sobre a pandemia da COVID-19 de acordo com a região geográfica de localização. 2020.

\begin{tabular}{|c|c|c|}
\hline \multirow[b]{2}{*}{ Características dos grupos de pesquisa } & \multicolumn{2}{|c|}{ Quantidade de grupos } \\
\hline & $\mathbf{n}$ & $\%$ \\
\hline \multicolumn{3}{|l|}{ Região geográfica do Brasil } \\
\hline Sudeste & 21 & 45,65 \\
\hline Nordeste & 11 & 23,91 \\
\hline Centro Oeste & 8 & 17,39 \\
\hline Sul & 5 & 10,87 \\
\hline Norte & 1 & 2,17 \\
\hline
\end{tabular}

Na Tabela 2 são apresentados os tipos de instituição e as áreas/subáreas de vinculação dos grupos de pesquisa. A maioria correspondeu a instituição pública. Houve o predomínio dos grupos da área/subárea das Ciências da Saúde/Medicina e das Ciências Biológicas/Microbiologia.

Tabela 2. Descrição dos grupos de pesquisa sobre a pandemia da COVID-19 de acordo com o tipo de instituição e grande área/subárea de vinculação. 2020.

Características dos grupos de pesquisa

Quantidade de grupos

Tipo de instituição

Pública

Privada

Grande área/subárea

Ciências da Saúde/Medicina

Ciências Biológicas/Microbiologia

Ciências Exatas e da Terra/Química

Ciências Sociais Aplicadas/Direito

Ciências Biológicas/Parasitologia

Ciências Biológicas/Farmacologia

Ciências Biológicas/Biotecnologia

Ciências Biológicas/Imunologia

Ciências Biológicas/Ecologia

Ciências Sociais Aplicadas/Administração

Ciências da Saúde/Fisioterapia e Terapia ocupacional 


\begin{tabular}{lll}
\hline Ciências da Saúde & 1 & 2,17 \\
\hline Ciências da Saúde/ Saúde coletiva & 1 & 2,17 \\
\hline Ciências Humanas/Ciências Política & 1 & 2,17 \\
\hline Ciências Exatas e da Terra/Ciências da Computação & 1 & 2,17 \\
\hline Ciências Humanas/Sociologia & 1 & 2,17 \\
\hline Ciências Exatas e da Terra/Matemática & 1 & 2,17 \\
\hline Ciências Sociais Aplicadas/Economia & 1 & 2,17 \\
\hline Ciências da Saúde/Farmácia & 1 & 2,17 \\
\hline Ciências Sociais Aplicadas/Turismo & 1 & 2,17 \\
\hline Engenharias/Engenharia Sanitária & 1 & 2,17 \\
\hline Ciências Biológica/Biotecnologia & 1 & 2,17 \\
\hline Ciências da Saúde/Saúde coletiva & 1 & 2,17 \\
\hline
\end{tabular}

Na Tabela 3 são apresentadas as informações sobre as linhas de pesquisa, total e específicas para COVID-19. Houve o predomínio de grupos de pesquisa com quatro $(21,74 \%)$ e três linhas $(17,39 \%)$ no total. Sobre as linhas de pesquisa especificas, a maioria apresentou uma linha sobre o tema da COVID-19 $(84,78 \%)$
Na Tabela 4 são descritos os conteúdos extraídos dos objetivos das linhas de pesquisa específicas para a pandemia da COVID-19. Houve o predomínio de conteúdos relacionados ao tratamento farmacológico e diagnóstico. Em cinco linhas específicas não constava a descrição dos respectivos objetivos.

Tabela 3. Descrição da quantidade de linhas de pesquisa no total e específicas para a pandemia da COVID-19. 2020.

\begin{tabular}{lcc}
\hline \multirow{2}{*}{ Informações } & Quantidade de grupos \\
\hline Quantidade de linhas de pesquisa no total dos grupos de pesquisa & $\mathbf{n}$ & $\mathbf{\%}$ \\
\hline $\mathbf{1}$ linha & 6 & 13,04 \\
$\mathbf{2}$ linhas & 5 & 10,87 \\
$\mathbf{3}$ linhas & 8 & 17,39 \\
$\mathbf{4}$ linhas & 10 & 21,74 \\
$\mathbf{5}$ linhas & 2 & 4,35 \\
$\mathbf{6}$ linhas & 1 & 2,17 \\
$\mathbf{7}$ linhas & 4 & 8,70 \\
$\mathbf{8}$ linhas & 2 & 4,35 \\
$\mathbf{9}$ linhas & 2 & 4,35 \\
$\mathbf{1 2}$ linhas & 2 & 4,35 \\
$\mathbf{1 3}$ linhas & 2 & 4,35 \\
$\mathbf{1 6}$ linhas & 1 & 2,17 \\
$\mathbf{2 1}$ linhas & 1 & 2,17 \\
Quantidade de linhas de pesquisa específicas dos grupos de pesquisa & & \\
$\mathbf{1}$ linha & 39 & 84,78 \\
$\mathbf{2}$ linhas & 4 & 8,70 \\
$\mathbf{3}$ linhas & 2 & 4,35 \\
$\mathbf{4}$ linhas & 1 & 2,17 \\
\hline
\end{tabular}


Tabela 4. Descrição dos conteúdos extraídos dos objetivos das linhas de pesquisa específicas para a pandemia da COVID-19. 2020.

\begin{tabular}{lcc}
\hline Conteúdo dos objetivos das linhas de pesquisa específicas & Frequência absoluta & Frequência relativa \\
\hline Tratamento farmacológico & 9 & 16,98 \\
Diagnóstico & 8 & 15,09 \\
Epidemiologia & 7 & 13,21 \\
Informação e comunicação & 7 & 13,21 \\
Saúde ocupacional & 5 & 9,43 \\
Economia & 5 & 9,43 \\
Tratamento não-farmacológico & 4 & 7,55 \\
Engenharia e medidas de biossegurança & 3 & 5,66 \\
Saúde coletiva & 3 & 5,66 \\
Sustentabilidade ambiental & 1 & 1,89 \\
Processos de ensino-aprendizagem & 1 & 1,89 \\
\hline
\end{tabular}

\section{DISCUSSÃO}

A pandemia da COVID-19 gerou o fenômeno do novo "boom" da produção científica, observando-se um fluxo contínuo e crescente de artigos, em diferentes áreas, no que diz respeito aos seus impactos sociais, econômicos, geográficos, culturais e principalmente voltados a saúde pública. Esse fator denota o compromisso da comunidade científica, da área das ciências e tecnologias, em direcionar esforços na busca do melhor caminho para o enfrentamento da pandemia, seja durante e/ou após o pico da curva de infecção pelo vírus.

Atualmente, o Brasil conta com a ajuda de 46 grupos de estudos com linhas de pesquisas específicas para investigar a pandemia da COVID-19, formados por aproximadamente 1.093 pesquisadores, professores doutores, alunos de pós-graduação e profissionais técnicos. Entre os grupos do diretório do CNPq, o mais antigo foi criado no ano de 1969 , e somente no ano de 2020 foram registrados mais 15 novos grupos de pesquisa, representando aproximadamente um terço dos 46 grupos identificados. Esses dados refletem que a nova emergência abriu espaço para o avanço da ciência no atendimento a situações de emergência global em saúde. A pesquisa realizada por Ribeiro et al. (2020), destacou que 16 estudos de intervenção sobre COVID-19 foram registrados na Comissão Nacional de Ética em Pesquisa (CONEP) e seguem em andamento. Tais resultados evidenciam também que a ciência brasileira continua resistindo, mesmo em meio as crises orçamentárias e políticas que reverberam na falta de apoio e incentivo a pesquisa nacional (VENTURA et al., 2020).

Embora exista um número de grupos de pesquisa superior ao número de estados brasileiros, percebe-se que houve o destaque para a região sudeste do país, como observado em outras publicações sobre grupos de pesquisa (BARATA, 2008; CRUZ; OLIVEIRA, 2019). Concomitantemente, esta região também compõe o estado de São Paulo, que representa local de destaque pela quantidade de casos da COVID-19 no país, ao mesmo tempo em que sedia agências de fomento à pesquisa historicamente consolidadas, como a FAPESP (Fundação de Amparo à Pesquisa do Estado de São Paulo). Destacaram-se também os grupos vinculados as regiões dos estados do Rio de Janeiro e Minas Gerais. No Centro-Oeste destaca-se o Distrito Federal como polo das pesquisas sobre COVID-19 na região.

O total de 95,65\% dos grupos de pesquisa foram provenientes de instituições públicas, logo, essas permitem o acesso gratuito aos estudos realizados ao longo da pandemia, o que por sua vez é essencial na socialização do conhecimento que qualifica a tomada de decisão, seja na área clínica, gestão pública, vigilância em saúde ou sanitária (MOTA; FERREIRA; LEAL, 2020). É válido destacar que a maioria das decisões e ações do Ministério da Saúde, bem como o decreto de reativação do Grupo Executivo Interministerial de Emergência em Saúde Pública de Importância Nacional e Internacional (GEI-ESPII), cuja atribuição é a articulação de medidas de preparação e enfrentamento de emergências em Saúde Pública, baseiam-se em estudos 
realizados por instituições de ensino públicas brasileiras (CRODA; GARCIA, 2020). Não se pode negar que as respostas mais imediatas e adequadas a pandemia dependem, sem dúvida, da existência dos sistemas de saúde bem estruturados, de amplo acesso, preventiva, com capacidade de vigilância, cuidado, tratamento e pesquisa conduzidas por profissionais com competência técnica, científica, ética e humanística. Portanto, a valorização da produção científica e tecnológica, a intervenção na estrutura social, o financiamento da formação acadêmica pública, distribuição da força de trabalho concentrada nos centros urbanos, e retomada de investimento no Sistema Único de Saúde não podem ser negligenciados pelas autoridades públicas (RAFAEL et al., 2020).

Quanto aos objetivos das linhas de pesquisa específicas, o estudo apontou predominância em relação ao diagnóstico, tratamento farmacológico e epidemiologia, e mecanismos de comunicação e informações. Esta tendência pode ser compreendida pelo fato destes desfechos formarem os principais pilares de combate a COVID-19. Dessa forma, diagnosticar com agilidade e eficácia os casos suspeitos é o alicerce para condução clínica e proteção da vida e saúde coletiva. Sendo assim, o mapa da situação real de saúde no que tange a COVID-19 só se torna possível com a testagem e confirmação precisa dos casos, como por exemplo, com base a coleta de materiais respiratórios (LIMA, 2020). Não obstante, o diagnóstico é um instrumento que viabilizará a condução de estudos epidemiológicos que permitam compreender a evolução da doença em nível territorial e a partir disso, planejar ações de combate. $\mathrm{O}$ diagnóstico possibilita da avaliação das ações de contingenciamento, a exemplo, do isolamento social ou "lockdown" (PRADO et al., 2020).

Por outro lado, é preciso destacar a necessidade de uma agenda de pesquisa permanente que considere não apenas "a evolução de doenças específicas, mas o impacto das crises sobre a saúde das populações, além da investigação das causas sociais, ambientais, econômicas e políticas das epidemias" (VENTURA et al., 2020). A ênfase em pesquisas na busca de vacinas e outras tecnologias, assim como a necessidade de construção de sistemas internacionais de vigilância capazes de oferecer uma resposta imediata e pontual à contenção das epidemias é sem dúvida relevante. Entretanto, a produção de conhecimento deve exceder a perspectiva puramente tecnológica e biomédica das emergências, levando em conta questões macroestruturais dos processos de saúde/doença e sua determinação social, o que ressalta o valor das ciências humanas e sociais (VENTURA et al., 2020).
Diante desse panorama da ciência brasileira em torno da COVID-19, é notório que ainda caminhando em direção ao desconhecido, os grupos de pesquisa tendem a expandir suas linhas de pesquisa, afim de contribuir para a prevenção e o controle da COVID-19 e doenças do gênero. Novas subáreas devem se expandir e se destacar nos estudos, à medida que a doença passa a ser compreendida e, até mesmo, após a queda da curva de infecção pelo vírus, o que deverá permitir reflexões quanto ao plano de estudos emergenciais para serem realizados por pesquisadores de instituições de ensino e pesquisa, públicas e/ou particulares. Ademais, as investigações poderão iluminar que o sofrimento gerado pela pandemia não se deve exclusivamente a virulência do coronavírus, mas as condições que acentuam a gravidade e o enfrentamento da pandemia como a desigualdade social, os interesses econômicos e políticos e as ações do poder público acima das necessidades de saúde da população. Como apontado por Grisotti (2020), as medidas oficiais do governo para controle da curva epidemiológica de casos da COVID-19 trazem à tona os profundos impactos psicológicos, sociais e econômicos, gerando a necessidade de uma nova agenda de pesquisa.

Este estudo apresenta algumas limitações no que se refere a possibilidade de subregistro das informações, uma vez que alguns grupos de pesquisa que estão desenvolvendo estudos sobre a COVID-19 podem não ter realizado a atualização dos seus cadastros no diretório do $\mathrm{CNPq}$ ou não incluíram ou adequaram suas linhas temáticas para contemplarem o tema. Outrossim, a constante progressão da pandemia ao longo do tempo pode favorecer o encaminhamento de outros objetivos de investigação, bem como a mudança da ênfase estabelecida até o presente momento.

Com base nessas considerações, o estudo apresentou um panorama dos grupos de pesquisas brasileiros que tem se dedicado em desenvolver estudos, fundamentar as políticas públicas e as intervenções na sociedade para enfrentamento da COVID-19. A maior concentração de estudos nas localidades de maior desenvolvimento econômico e histórico em pesquisa, mostra que a migração da ciência e tecnologia para as regiões brasileiras ainda é perene e paulatina. As linhas de pesquisa direcionadas à investigação da COVID-19 mostram que as produções se concentram na área da saúde, mas englobam também aspectos econômicos, sociais e educacionais. Recomenda-se que estudos adicionais abordem questões como o impacto da produção científica brasileira sobre a COVID-19 nas evidências cientificas sobre o tema, bem como os registros de cooperações interinstitucionais das instituições nacionais. 


\section{REFERÊNCIAS}

BARATA, R. B. A Pós-Graduação e o campo da Saúde Coletiva. Physis: Revista de Saúde Coletiva, v. 18, n. 2, p. 189-214, 2008.

BARDIN, L. Análise de conteúdo. Lisboa, Portugal: Edições 70; 2002.

BRASIL. Brasil.io COVID-19: Boletins informativos e casos do coronavírus por município por dia [25 de julho de 2020]. Disponível em: https://brasil.io/dataset $/$ covid19/caso/?search $=\&$ date $=202$ $0-02-$

$25 \&$ state $=\&$ city $=\&$ place_type $=\&$ is_last $=\&$ city_ibge_cod $\mathrm{e}=\&$ order_for_place $=$

CATTANI, M. Global coalition to accelerate COVID-19 clinical research in resource-limited settings. The Lancet, v. 395, n. 10233, p. 1322-1325, 2020.

CHAVES, A. O.; CRUZ, G. P. Eficiência do instituto federal baiano: análise dos grupos de pesquisa e propriedade industrial. Cadernos de Prospecção, v. 10, n. 3, p. 438-447, 2017.

CRODA, J. H. R.; GARCIA, L. P. Immediate health surveillance response to COVID-19 epidemic. Epidemiologia e Serviços de Saúde, v. 29, n. 1, p. e2020002, 2020.

CRUZ, M. M. DA.; OLIVEIRA, S. R. A. Grupos de pesquisa de avaliação em saúde no Brasil: um panorama das redes colaborativas. Saúde em Debate, v. 43, n. 122, p. 657-667, 2019.

DIAS, G. P. et al. Scientific Production of Researchers from the Brazilian Council for Scientific and Technological Development (CNPq) in the Neuroscience area. Revista Brasileira de Educação Médica, v. 44, n. 2, p. e049, 2020.

FAPESP. A pesquisa científica sobre coronavírus. Disponível em: <https://revistapesquisa.fapesp.br/apesquisa-cientifica-sobre-coronavirus/>. Acesso em: 25 jul. 2020.

GRISOTTI, M. Pandemia de Covid-19: agenda de pesquisas em contextos de incertezas e contribuições das ciências sociais. Physis: Revista de Saúde Coletiva, v. 30, n. 2, p. e300202, 2020.

LIMA, C. M. A. DE O. Informações sobre o novo coronavírus (COVID-19). Radiologia Brasileira, v. 53, n. 2, p. 5-6, 2020.

MACÁRIO, E.; REIS, L. F. Covid-19-divida-publica-efinanciamento-de-CT-Versão-Final. Disponível em: $<$ https://www.abrasco.org.br/site/wpcontent/uploads/2020/04/Covid-19-divida-publica-efinanciamento-de-CT-Versão-Final.pdf >. Acesso em: 25 jul. 2020.

MENDONÇA, T. C. F.; SOUSA, T. F.; BAHIA, C. S.; COELHO, F. T. Caracterização dos grupos de pesquisa da área da educação física do brasil que estudam a deficiência. Revista Brasileira de Ciências da Saúde, v. 21, n. 4, p. 313-322, 2017.

MOTA, D. M.; FERREIRA, P. J. G.; LEAL, L. F. Produção científica sobre a Covid-19 no Brasil: uma revisão de escopo. Visa em Debate, 18 maio 2020. Disponível em: <https://visaemdebate.incqs.fiocruz.br/index.php/visaem debate/article/view/1599>. Acesso em: 25 jul. 2020.

NUNES, J. The COVID-19 pandemic: securitization, neoliberal crisis, and global vulnerabilization. Cadernos de Saúde Pública, v. 36, n. 5, p. e00063120, 2020.

OPAS. Pandemia COVID-19. [25 de julho de 2020]. Disponível

em:

https://www.paho.org/bra/index.php?option=com_conten $\mathrm{t} \&$ view=article\&id=6120:oms-afirma-que-covid-19-eagora-caracterizada-como-pandemia\&Itemid $=812$

PRADO, M. F. et al. Análise da subnotificação de COVID-19 no Brasil. Revista Brasileira de Terapia Intensiva, v. 32, n. 2, p. 224-228, 2020.

RAFAEL, R. M. R. et al. Epidemiologia, políticas públicas e pandemia de Covid-19: o que esperar no Brasil? Revista Enfermagem UERJ, v. 28, p. 49570, 2020.

RIBEIRO, T. B.; MAZOTTI, T. A.; NOBRE, R. R. C.; STEIN, A.; MELO, D. O. Iniciativas brasileiras de estudos de intervenção em andamento para a COVID-19. [14 abr. 2020]. Disponível em: $<$ https://preprints.scielo.org/index.php/scielo/preprint/vie w/45>. Acesso em: 25 jul. 2020.

SCHMITT, B. D.; BERTOLDI, R.; MAZO, J. Z. Grupos de pesquisa em ciências da saúde e o uso da história oral. Arquivos de Ciências da Saúde, v. 24, n. 2, p. 9-13, 
2017.

VENTURA, D. F. L. et al. Desafios da pandemia de COVID-19: por uma agenda brasileira de pesquisa em saúde global e sustentabilidade. Cadernos de Saúde Pública, v. 36, n. p. e00040620, 2020.

WANG, C. et al. A novel coronavirus outbreak of global health concern. The Lancet, v. 395, n. 10223, p. 470-473, 2020 .

WHO. "Solidarity" clinical trial for COVID-19 treatments. Disponível em:

https://www.who.int/emergencies/diseases/novelcoronavirus-2019/global-research-on-novel-coronavirus2019-ncov/solidarity-clinical-trial-for-covid-19-

treatments >. Acesso em: 25 jul. 2020.

ZHU, N. et al. A Novel Coronavirus from Patients with Pneumonia in China, 2019. The New England Journal of Medicine, v. 382, n. 8, p. 727-733, 2020. 